\title{
Cours de formation en radioprotection et en technique radiologique destinés aux médecins non radiologues
}

J. Marti

\begin{abstract}
Objectifs
Au terme de l'ordonnance du 22 juin 1994 sur la radioprotection ( $\mathrm{ORaP})$, il est exigé, à l'article 18 , que les médecins désirant obtenir une autorisation pour l'exploitation d'une installation à rayons X à usage médical possèdent, pour exercer leur fonction d'expert local de radioprotection, "une formation en radioprotection et en technique radiologique sanctionnée par un examen et reconnue par l'Office fédéral de la santé publique (OFSP)».

Pour les médecins ayant fait leur demande d'autorisation après la date de mise en vigueur de l'ordonnance ( $1^{\text {er }}$ octobre 1994), une formation théorique et pratique d'une semaine est prévue.

Les cours présentés par la Fondation PRORAME correspondent aux exigences de l'OFSP et sont reconnus.
\end{abstract}

\section{Inscription}

Condition d'admission

Le diplôme fédéral de médecine, ou une formation étrangère équivalente et reconnue.

\section{Programmes détaillés}

Les programmes détaillés (avec formulaire d'inscription) peuvent être obtenus sur demande à l'Institut de radiophysique appliquée, Grand-Pré 1, 1007 Lausanne.

\section{Délai d'inscription}

Deux mois avant le cours; l'inscription sera confirmée par écrit.

\section{Correspondance:}

Jürg Marti

OFSP, Division Radioprotection

Service formation en radioprotection

CH-3003 Berne

Tél. 0313229613

E-mail: juerg.marti@bag.admin.ch
Finance d'inscription

La finance d'inscription est de Fr. 1800.-; elle couvre l'enseignement et la documentation distribuée. L'entretien (repas, hôtel) et les déplacements ne sont pas compris dans ces prestations.

\section{Lieux et dates}

Les dates et lieux des prochains cours pour la Suisse romande sont les suivants:

\begin{tabular}{lll}
\hline Institution & Dates & Lieux \\
\hline Fondation PRORAME & 8.7.-12.7.2002 & Lausanne \\
\hline & $9.12 .-13.12 .2002$ & Genève \\
\hline
\end{tabular}

Justification de la compétence d'expert pour les médecins qui disposaient d'une autorisation d'exploiter une installation radiologique avant le $1^{\text {er }}$ octobre 1994

Les médecins qui ont reçu une autorisation pour une installation radiologique avant le $1^{\text {er }}$ octobre 1994, sont considérés jusqu'au 30 septembre 2004 comme experts. Si l'installation radiologique est encore utilisée après cette date, la compétence d'expert doit être justifiée. Elle peut être obtenue par la fréquentation du cours de 5 jours mentionné ci-dessus ou en se présentant à un examen centralisé. Les informations concernant cet examen seront publiées dans le bulletin de l'OFSP et dans le journal des médecins suisses.

\section{Information}

Des informations complémentaires peuvent être obtenues à l'adresse suivante: Institut de radiophysique appliquée, Grand-Pré 1, 1007 Lausanne, tél. 021 62334 34, fax 0216233435. 